\title{
IS SUBJECTIVE APPEARANCE ALTERED BY VERBAL INFORMATION? CHILDREN'S UNDERSTANDING OF COLORBLINDNESS
}

\author{
Monica Wengrowicz COOPER ${ }^{1)}$, Rick GRIFFIN ${ }^{2)}$ \\ ${ }^{1)}$ Boston College, U.S.A., ${ }^{2)}$ Tufts University, U.S.A. \\ and \\ Ellen WINNER ${ }^{1)}$ \\ ${ }^{1)}$ Boston College, U.S.A.
}

\begin{abstract}
We investigated children's understanding that visual perception is subjective and not alterable by propositional information. Thirty-six children (3.4 to 6.8 years) were tested in a screen condition (in which a puppet views a colored toy through a differently colored screen), and a colorblind condition in which a puppet (introduced as colorblind) looks directly at the toy. Asked to name the color of the object that the puppet sees, children under four fail in both conditions, revealing difficulty grasping false perception. The puppet was then told the correct color of the toy by $\mathrm{E}$, and the child was again asked to name the color the puppet sees. Even the oldest children failed this question in the colorblind condition, revealing the misconception that a label can alter a color's perception. Children as old as six do not understand that although verbal information is an originator of belief, it cannot alter perception.
\end{abstract}

Key words: theory-of-mind, understanding visual perception

At the core of the idea of subjectivity lies the incontrovertible truth that we can never know how the world is perceived and experienced by others. When you call something red, does its redness look to you like it looks to me? There is no way to answer this question. Colorblindness is an extreme case of subjective perception. The exercise of trying to understand how the world looks to a colorblind person serves to particularly underscore the idea of subjective experience.

A critical test of our understanding that every person's experience of color is subjective is the recognition that we cannot come to a shared experience of color by agreeing on verbal labels. That is, teaching a colorblind person the correct color terms for various colors will not alter that person's subjective experience of the color.

A fully developed theory of mind (Perner, 1992) must allow for an understanding of the subjective experience of color, and for the understanding that we cannot "correct" another's differing perception of color through verbal labels. How children make sense of colorblindness is an aspect of their theory of mind that has not, to our knowledge, been

We thank the children, parents and administrators of the Arlington Children Center in Arlington, Massachusetts and, in particular, Barbara O’Neal, the Center's Educational Director.

Correspondence concerning this article should be addressed to Monica Wengrowicz Cooper, c/o Ellen Winner, Psychology Department, Boston College, Chestnut Hill, MA 02467, U.S.A. (e-mail: Monica_Cooper@post.Harvard.edu). 
studied. Yet such study can tell us something important about children's theory of mind: specifically, when do children understand that perception is one route to knowledge, while language is another route, and verbal labels cannot alter how the world is perceived?

Researchers have investigated children's understanding that an object which appears one color to the child may appear another color to someone else, but they have not gone on to determine how children understand the source of this difference and its possibilities for correction. In a series of experiments, Flavell, Green and Flavell (1986) studied how children as young as three distinguish between the appearance and the reality of an object's color. In one of those experiments (Study 4), the researchers compared the performance of children (age 3.1 to 4.2 years) in a "Level 2 perceptual perspectivetaking" task to that in an "appearance-reality" task by means of similar tests involving the use of a colored screen. Level 2 perceptual perspective-taking refers to the understanding that the same object looks different when seen from different perspectives. For the perspective-taking experiment, the child was first shown a cutout of a pink seal directly and then through a blue screen (Flavell et al., 1986). The child was told that the seal looks blue to the child because of the screen but it looks pink to the experimenter, who was on the other side of the screen. The seal was then moved to the child's side of the screen and the child was told that the seal looks pink to the child but blue to the experimenter. The perspective-taking test consisted of the experimenter showing the child a new object, placing it on the experimenter's side of the screen and asking, "You are looking at the X with your eyes right now. Does it look A (e.g., blue) or does it look B? I am looking at X with my eyes right now. Does it look A to me or does it look B to me?" The questions asked regard only appearance: they were always phrased with the use of the verb "look." Children who realized that the object looked different to self and other passed this task.

For the appearance-reality test conducted in the same session, the child was pretrained and tested in the same way as in the perspective-taking task except that instead of being asked how the object looked to the experimenter, the child was asked a reality question: "What color is the X really and truly? Is it really and truly A or is it really and truly B?" The answer to the reality question was always the same as the answer to the appearance-for-other question because the object was always placed on the experimenter's side of the screen. Children succeeded in this task if they were able to encode two representations of the object, one its appearance and the other one its reality.

Flavell et al. (1986) concluded that appearance-reality and Level 2 perspectivetaking abilities are similar and appear at the same time. However, a close look at the color perception results reveals that children performed better on the perspective-taking than on the appearance-reality task. In fact, a majority of the children passed the color perspective-taking task but a majority failed the color appearance-reality. The results of Flavell et al. (1986) suggest that representing two appearances, one for self and one for other, is easier for young children (when what is at issue is the color of an object) than representing an object's appearance and its real identity. Perhaps children at an early age discover that an object's color is amenable to change (due to factors such as light or the atmosphere around it) and thus realize that it is not unusual for an object to appear different in color to two different observers. 
In Flavell et al. (1986), the object's color appears different to the child because the child views it through a colored screen. The child can experience looking at the object directly and then through the screen and can thus, through memory and inference, know how someone else would see the object when looking at it directly or through the screen. However, children might pass this test in one of two ways: they can pass because they genuinely understand that the experience of color is subjective and can be false; or they can pass because they believe, incorrectly, that the screen placed in front of the object actually modifies the physical surface of the object itself temporarily (something akin to painting it on one side). And thus, although the child may succeed in ascribing one appearance for self and a different one for other, he can do so by placing the source of the difference in the object.

One way to determine whether children grasp the subjective nature of color perception is to examine their understanding of how a colorblind person sees the world. In the case of colorblindness, there is no external colored screen through which to view an object. Instead, colorblindness is as if the screen is internal. To understand that a colorblind person has a "false" perception of an object's color, and to understand that the way the colorblind person sees color cannot be "corrected" by providing him or her with verbal color labels, requires a genuine understanding of the subjective nature of visual experience. In the present study we investigated children's understanding of colorblindness in order to determine when children understand the subjective character of visual perception.

We examined children's understanding of conflicting views of an object's color in a condition like Flavell's, with an external colored screen, as well as in a new, colorblind condition in which children had to infer how a colorblind person sees an object's color. And we asked, in both conditions, whether providing the other person (looking through a screen, or colorblind) with the correct color label would change how the person saw the color. We hypothesized that the presence of an external screen should allow the child to infer that another person sees an object's color differently even if a child does no really understand the subjectivity of appearance. Thus, children should succeed in recognizing that a person looking through an external screen sees a yellow object as red before they succeed in recognizing that a colorblind person sees a yellow object as red. We also hypothesized that the presence of an external screen should make it easier to understand that simply providing the correct labels will not alter the other's perception of the object's color. This is because the child has had the chance to experience looking through the screen and can thus infer that knowing the correct color label will not change the perception of the object's color through the screen. However, in the case of colorblindness, the child has no relevant experience, and should be more likely to think that providing appropriate labels should be all that is needed. Such a finding would demonstrate that children do not really understand how the different informational modalities of perception and communication interact with each other and with belief. The question of whether a person's perception can be altered by providing correct color labels was the critical test of children's understanding of the informational sources of perception and communication. Children can only be credited with understanding visual perception and its subjective 
nature if they recognize that a red object that looks green continues to look green even after the viewer is told that the object is red.

The study reported here is related to research on children's attributions of false belief since seeing and verbal information are both causal origins of knowledge and belief. Wimmer, Hogrefe, and Sodian (1992) claim that before the age of four, children do not know where their knowledge comes from. This conclusion follows an experiment in which children sat in front of an unmarked box and were either allowed to look into the box or the contents of the box were whispered in their ear. Children were then asked if they knew what was in the box and how they knew it. Next they witnessed another child sitting across from them either looking into the box or being told what was in the box. They were asked whether the other child knew what was in the box and how the other child knew what was in the box (Wimmer, Hogrefe \& Perner, 1988). Although children use perception and verbal communication as sources of belief from the first year of their lives, they do not realize that these are the source of their beliefs. Before the age of four they may equate seeing with knowledge or apply a functional correlation between the two (O’Neill, Astington, \& Flavell, 1992; but see Pratt \& Bryant, 1990). However, they fail to understand the origins of their own knowledge, and consequently, they also fail to understand what another person knows (O’Neill et al., 1992; Wimmer et al., 1992).

Wimmer et al. (1992) speculate that children younger than four may fail the false belief task for two possible reasons. (1) They may not understand the informational origins of belief. (2) They do not have "representational complexity" in place. That is, they do not understand that the other holds an incorrect representation as correct at the time the child holds the correct one (as opposed to pretense play in which the child knows the incorrect representation to be not true). Wimmer et al. (1992) point out that young children fail in the attribution of false belief not because they cannot handle representational complexity but because of a lack of understanding of informational origins. This conclusion derives from the fact that children acquire false belief attribution earlier than they do inferential attribution. In both these tasks, an understanding of informational origins is necessary, but only false belief attribution demands representational complexity. Given that false belief attribution is easier and develops earlier than inferential attribution, these researchers conclude that young children's failure at false belief attribution is due to a failure in understanding informational origins. Our study examines children's emergent understanding of the informational sources of seeing and verbal labels, and the relationship between these two sources of knowledge (i.e., which one is dominant).

We examined children's understanding of the subjective nature of seeing and the primacy of seeing as a source of knowledge over verbal information. We confronted children with information provided by the channel of seeing which conflicted with information provided by verbal labels. We showed children, for example, a red car but told them that the car appeared gray to a colorblind puppet. We then asked the child whether the car would appear red to the puppet if the puppet were told that the car was "red". The question underlying this study was how children understand these two sources of information (seeing and verbal labels) and whether children realize that the provision of 
verbal labels that conflict with how something looks cannot alter how things look.

Our study is similar to other false belief studies in that to respond correctly the child has to understand that the other holds an incorrect representation at the time that the child holds the correct one. Also, the child has to understand the primacy of visual perception over communicated information - that the information conveyed by seeing cannot be changed by communicated information. By investigating children's understanding of the informational sources of belief (i.e. perception and verbal information) we hoped to add to our understanding of why children younger than four fail at sorting these out in the false belief task (see also Gopnik \& Graf, 1988).

In short, we investigated when children understand the difference between knowing (or believing), on the one hand, and seeing, on the other hand. That is, when do children realize that visual perception and linguistic information are both routes to belief, but that linguistic information cannot alter the phenomenology of visual perception? And does this realization come more readily to children when the source of the "incorrect" perception is external (i.e., a colored screen) than when it is internal (i.e., a colorblind puppet)?

\section{METHODS}

\section{Participants:}

Participants were thirty-six children (21 boys and 15 girls) from a primarily white middle class suburban day care center, ranging in age from 3.4 to 6.8 years. All children were tested individually in one session in a familiar room of the day care center by two researchers.

\section{Procedure:}

Each child was tested in two conditions (order counterbalanced), each of which began with a training task to familiarize the children with the materials and task requirements. In the Screen condition, a puppet viewed a yellow and an orange watch through a red plastic box (making them both appear red), while the child saw them directly as yellow and orange. In the Colorblind condition, a puppet and the child viewed a red and a green car directly, but the puppet was said to be colorblind and unable to see the difference between red and green. Thus, in one condition the puppet cannot distinguish colors because of an external, visible obstruction, while in the other condition, the puppet cannot distinguish colors because of an internal, biological deficit.

Screen condition

Training. A red transparent plastic box was placed on its side on the table in front of the child with its opening away from the child. The child could look at the watches but could not take them out of the box. The box contained two toy watches, one orange and one yellow, next to one another. Viewed through the red box, the watches both appeared red. The child was asked, "See these two watches? Are they the same color or are they different colors? The correct answer was "same." (The words "different" and "same" were used in counterbalanced order for all questions in which they appear.) After the child said that the watches were the same color, the experimenter asked, "What color is this watch" for each one of the watches. The correct answer was "red." The experimenter then took the watches out of the box and said, "Now, look at the two watches! Are they the same color or are they different colors?" The correct answer was "different." The experimenter then asked again for the color of each watch. The correct answers here were "yellow" and "orange."

Perspective-Taking Question. Experimenter 1 (E1) placed the yellow and orange watches back inside the red transparent plastic box as the child observed while E1 commented, "We will put the yellow and orange watches back in the red box." The box was placed on its side, with its opening facing the child, so the 
child could see the actual color of the watches. A second experimenter (E2) brought in a puppet and introduced it to the child by saying, "This is Max." E2 sat opposite the child. E2 always spoke for the puppet while E1 talked to the child. E1 said to the child, "Now, when Max looks at these two watches, what does he see? Do they look to him like they are the same color or do they look to him like they are different colors?" The correct answer here was "same."

Labels Question. The experimenter then said, "Let's see what Max says. Max, see these two watches? Are they the same color or are they different colors?" The puppet then answered, "The same. They are both red." E1 then turned to the child and said, "Hey. He got it wrong!" E1 turned back to Max and said, "Max, I'm going to tell you what color each watch is. Max, this watch is orange and this watch is yellow. Now when you look at the watches, do they still look like the same color or do they look like different colors?" Then E1 asked, "What will Max say?" The correct answer here was "same" since verbal information about the real color of the watches should not alter the appearance of the watch.

Fix-it Question. E1 then said: "Now I am going to try something else to help Max." And then, turning to Max, E1 continued, "Max, I'm going to take away this box. Now, when you look at the watches do they still look like the same color or do they look like different colors?" E1 now faced the child and asked, "What will Max say?" This was the Fix-it Question. The correct answer here was "different."

Best Way Question. If the child responded that Max would see the watches as different colors after both kinds of aids (being provided with the correct color labels and being allowed to see the watches without looking through the box), E1 asked, "Which would be the very best way to make Max see that the watches are different colors, telling him the colors, or taking away the box?" The order of the options in this question was counterbalanced. The correct answer here was "taking away the box."

\section{Colorblind Condition}

Training. In the training phase of the Colorblind Condition, the child was shown a sheet of paper on which was drawn a red and a green circle. E1 asked the child, "See these two circles? Are they the same color or are they different colors?" After the child answered, the experimenter asked the child: "What color is this circle?" for each one of the two circles.

Perspective-Taking Question. A puppet was brought in by E2 and placed facing the red and green circles. E1 then said to the child, "This is Tommy. Let's ask Tommy if the circles are the same color or different colors." Then, turning to the puppet, E1 asked, "Tommy, see these two circles? Are they the same color or are they different colors?" The puppet replied, "The same. They are both gray." E1 then faced the child and said: "Hey, he got it wrong! I am going to tell you something about Tommy. Tommy doesn't see colors right. You see; he is colorblind. Have you ever heard of somebody being colorblind? Tommy is colorblind because he can't tell the difference between green and red. They both look gray to him." E1 then placed on the table a red car and a green car and asked the Perspective-Taking question: "Let's look at these two cars. Now, when Tommy looks at these two cars, what does he see? Do they look to him like they are the same color or do they look to him like they are different colors?" The correct answer, as in the Screen condition, was "same."

Labels Question. After the child answered, E1 said: "Let's see what Tommy says" and facing Tommy, E1 said, "Tommy, see these two cars?" Tommy responded affirmatively and E1 said, "Are they the same color or are they different colors?" Tommy responded, "The same. They are both gray." E1 said to the child: "Hey, he got it wrong. Is there anything we can do to make Tommy see that this car is green and this car is red?" E1 then turned to Tommy and said: "I'm going to tell you what color each car is. Tommy, this car (pointing to the red car) is red and this car (pointing to the green car) is green. Now, when you look at the cars, do they still look like the same color or do they look like different colors?" The experimenter then turned to the child and asked, "What will Tommy say?" This was the Labels Question. The correct answer, as in the Screen Condition, was "same."

Fix-it Question. After the child replied, E1 said to the child: "Now I am going to try something else to help Tommy". Turning to the puppet, E1 said, "Tommy, I am a doctor and I am going to fix your eyes so that you see colors like everyone else." E1 then pretended to put something in Tommy's eyes. "Now, when you look at these cars, do they still look like the same color or do they look like different colors?" E1 turned to the child and said: "What will Tommy say?" This was the Fix-it Question, and the correct answer, as in the Screen Condition, was "different."

Best Way Question. If the child responded that Tommy would see the cars as different colors after both kinds of aids (being provided with the correct color labels and having his eyes medically altered), E1 asked, 
"Which would be the very best way to make Max see that the cars are different colors, telling him the colors, or fixing his eyes." The correct answer was "fixing his eyes".

Scoring:

For the purpose of some of the analyses, participants were divided into two groups: those who were four years old or younger (hereafter referred to as the younger group, $N=11$ ), and those who were older than four (hereafter referred to as the older group, $N=25$ ). For each question, children received a score of 1 (correct) or 0 (incorrect).

Hypotheses:

Four hypotheses were tested:

Perspective-Taking Question. Hypothesis la: The younger children were expected to pass the Perspective-Taking question at a rate above chance in the Screen but not the Colorblind condition. This is because in the Screen condition the reason for the abnormal color perception is concrete and visible; in addition, in this condition children can themselves experience the altered color perception (by looking through the red box). In contrast, in the Colorblind condition, the reason for the abnormal color perception is invisible and cannot be experienced by the child.

Perpective-Taking Question. Hypothesis $1 b$ : The older children were expected to pass the PerspectiveTaking question equally well in both conditions.

The remaining two hypotheses are relevant only for those children who recognize that the puppet sees colors incorrectly.

Hypothesis 2: In the Screen condition, those children who recognize the puppet's incorrect color perception should realize that provision of a verbal label will not alter the perception. Children should realize that the verbal label cannot alter perception in the Screen condition because they themselves can look through the red box and experience the reality of the altered perception. In the Colorblind condition, this realization should be more difficult, and children should not be able to respond correctly to the Labels question at a rate significantly above chance. We reasoned that in the Colorblind condition, children cannot make themselves see the colors the way the puppet does and hence should be tempted to believe that the puppet's only problem is not having the right labels.

Hypothesis 3: All children were expected to pass the Fix-It question. However, when asked to choose between removing the obstruction (removing the screen or fixing eyes) vs. merely providing a correct verbal label as the best way to fix the puppet's vision, children should succeed in the Screen condition but not in the Colorblind condition.

\section{RESULTS}

To test Hypothesis 1a, we examined the number of children in the younger group passing the Perspective-Taking Question. Table 1 shows that out of 11 children, 5 passed in the Screen condition, and 6 passed in the Colorblind condition. Neither of these rates was above chance, as shown by a one-tailed binomial test, $p=.50$. Thus, contrary to Hypothesis 1a, younger children had as much difficulty with perspective taking in the Screen as the Colorblind condition.

To test Hypothesis $1 \mathrm{~b}$, we examined the number of children in the older group passing the Perspective-Taking Question. Table 2 shows that out of 25 children 19 passed in the Screen condition, and 23 passed in the Colorblind condition. A one-tailed binomial test showed that these rates were significantly above chance in both conditions (Screen,

\footnotetext{
${ }^{1}$ The authors realize that by pretending to be a doctor that could fix a puppet's colorblindness, they were suggesting to participants that colorblindness is curable. The authors acknowledge that they should have debriefed the children after the testing session.
} 
Table 1. Responses to the Perspective Taking Question by the Younger Group

\begin{tabular}{lcc}
\hline Condition & Pass & Fail \\
\hline Screen & 5 & 6 \\
Colorblind & 6 & 5 \\
\hline
\end{tabular}

Table 3. Responses to the Labels Question, Both Age Groups Combined

\begin{tabular}{lcc}
\hline Condition & Pass & Fail \\
\hline Screen & 18 & 6 \\
Colorblind & 19 & 10 \\
\hline
\end{tabular}

Table 2. Responses to the Perspective Taking Question by the Older Group

\begin{tabular}{lcc}
\hline Condition & Pass & Fail \\
\hline Screen & 19 & 6 \\
Colorblind & 23 & 2 \\
\hline
\end{tabular}

Table 4. Responses to the Fix-It Question, Both Age Groups Combined

\begin{tabular}{lcc}
\hline Condition & Pass & Fail \\
\hline Screen & 22 & 2 \\
Colorblind & 26 & 3 \\
\hline
\end{tabular}

$p=.007$; Colorblind, $p<.002$ ). Taken together, these results demonstrate that children four or younger cannot take the perspective of the puppet, irrespective of condition, while children above four can do so, again irrespective of condition.

To test Hypothesis 2, we examined responses to the Labels Question by children who had passed the Perspective-Taking Question in each condition. (We had to exclude those children who had failed the Perspective-Taking question, since the Labels question assumes an understanding that the puppet sees the colors incorrectly. We could not examine younger and older children separately because cells were too small after excluding those who failed the Perspective-Taking question.) Table 3 shows that in the Screen condition, 18 out of 24 children passed the Labels question, a rate significantly above chance ( $p=.01$, one-tailed binomial test). Thus, in the Screen condition, children realized that the provision of a label has no effect on perception. Although 6 of the children tested in the Screen condition were younger than four and thus in the category of having passed the Perspective-Taking question but having passed it at chance, still, the group of 24 passed the Labels question in the Screen condition at a significant rate. Table 3 shows that in the Colorblind condition, 19 out of 29 children passed the Labels Question. While more children passed than failed, this rate did not pass the conventional significance cut-off of .05 ( $p=.07$, one-tailed binomial test). These results show that, as predicted, recognition of the ineffectiveness of providing a verbal label was less readily understood in the Colorblind than the Screen condition. In addition, of the 23 children older than four tested in the Colorblind condition with the Labels question, 15 passed and 8 failed. A one-tailed binomial test of these results showed the number of children passing not to be significantly above chance $(p=.11)$. These results show that the older children had similar difficulty to the younger children in passing the Labels question in the Colorblind condition.

To check Hypothesis 3, we first determined that children who passed the PerspectiveTaking Question in each condition had no difficulty passing the Fix-it question. Table 4 
Table 5. Responses to the Best-Way Question, Both Age Groups Combined

\begin{tabular}{lcc}
\hline Condition & Pass & Fail \\
\hline Screen & 15 & 5 \\
Colorblind & 18 & 5 \\
\hline
\end{tabular}

Note: 4 children in the Screen condition and 6 children in the Colorblind condition failed to provide a clear answer to the Best Way question and were thus not included in the analysis for this question.

shows that 22 out of 24 children passed the Fix-it question in the Screen condition ( $p<.001$, one-tailed binomial test), and 26 out of 29 passed the Fix-it question in the Colorblind condition ( $p<.001$, one-tailed binomial test).

We next examined responses to the Best Way question, when children had to choose between removing the obstruction (internal or external) or providing a correct label. Table 5 shows that 15 out of 20 children passed the Best Way question in the Screen condition $(p=.02)$, and 18 out of 23 children passed this question in the Colorblind condition $(p=.005)$. Four children in the Screen condition and 6 children in the Colorblind condition failed to give a clear answer to this question and were thus not scored.

The finding that children passed this question at a rate above chance in the Screen condition is consistent with Hypothesis 3. However, the finding that children also passed this question at a rate above chance in the Colorblind condition is inconsistent with Hypothesis 3. Once children were given a better alternative to providing a verbal label as a means of altering the puppet's perception, they recognized the alternative as better, whether in the Screen or the Colorblind condition. Thus, children's difficulty understanding the subjective nature of colorblindness is most clearly demonstrated in their response to the Labels question, but not to the easier Fix-it and Best Way questions.

\section{DISCUSSION}

In this study we used children's understanding of colorblindness to test children's understanding of two properties of visual perception: subjectivity and encapsulation. Visual perception is subjective because it is based on each individual's visual system and is tied to that system's peculiarities. Visual perception is encapsulated because a belief formed from visual information cannot be altered by verbal information. We are aware that verbal context can sometimes lead to visual illusions and hence there are cases in which verbal information does lead people to mis-see. However, in this study we examined situations in which verbal information should not be able to lead people to mis-see.

We examined children's understanding of visual experience in two situations. The situation of primary interest was the condition of colorblindness. Here, there is no external cue to allow the child to understand the other's visual perception. There is no way that the child can "try out" the colorblind person's experience of color. The comparison situation was that of looking at the world through a colored screen. Here, 
there is an external cue (the screen) that allows the child to experience the way that the other, looking through the screen, sees the world. We expected that the possibility of trying out another's view of the world would allow a clear understanding of the subjectivity and encapsulation of visual perception. The stringent test of an understanding of the subjectivity and encapsulation of visual perception is in the situation in which there is no possibility of trying out another's view of the world - and this was the situation afforded by the concept of a colorblind puppet.

The results presented here demonstrate, first of all, that children four or younger do not recognize the subjectivity of visual experience, as evidenced by their failure on two kinds of Level 2 perspective-taking tasks. They did not recognize that a puppet looking through a colored screen cannot see the "true" color of an object (Screen condition), nor did they recognize that a colorblind puppet cannot see the "true" color of an object (Colorblind condition). Both conditions proved equally difficult. However, children older than four passed both tasks equally well, demonstrating an understanding of the subjectivity of visual experience. They realized that a yellow watch looks red to a puppet looking at the watch through a red screen, and that a red car looks gray to a colorblind puppet.

We had hypothesized that children younger than four would pass the Level 2 perspective-taking test in the Screen condition because they themselves had first experienced looking through the screen. However, this hypothesis was not supported, and performance was as poor in the Screen condition as in the Colorblind condition. One interpretation of our results is that children four or younger do not understand that two people can look at the same object and each see it as a different color. Another interpretation is that failure on our task is not due to a difficulty in holding in mind two representations. Rather, failure could be due to the faulty belief that color is an immutable feature of a given object. That is, to see an object is to see its true color, just as to see an object is to see its true shape (see also Taylor \& Flavell, 1984).

However, findings from Flavell, Green, and Flavell (1986, Study 4) cast doubt on both of these interpretations. They reported that children younger than four could pass a Level 2 perspective-taking task that also involved looking through a colored screen. However, in their study, the experimenter looked directly at the object (and thus had the correct perception) while the child looked through the screen (and hence had the incorrect perception). It may be easier for the child to hold in mind both a correct and an incorrect view of the object when the incorrect one is the one presented to the child's own senses at the time of test. To pass such a test, the child must realize that his/her view is incorrect, and the salience of the "true" color may allow the child to reply correctly. In our study, the child must report the incorrect color and suppress the correct color. Taken together, the results of Flavell et al. (1986) and the present results suggest that children under four can hold two representations of color appearance in certain conditions, i.e. when they are looking at the incorrect one while the other person is looking at the correct one. However, in a more challenging situation, i.e. when they are looking at the correct appearance, they fail to represent the other person's (incorrect) view.

Our results also demonstrate that children do not understand the encapsulated nature 
of visual perception, as revealed by their performance on the Labels question. This question was only asked of those children who understood that the puppet saw the object's color incorrectly. To these children, we suggested telling the puppet what the correct color name was and asked them whether the puppet now saw the color correctly. Do children realize that provision of the verbal label cannot alter the subjective perception of the color for the puppet?

Children were far more likely to understand this challenging concept in the Screen than in the Colorblind condition. They understood that the puppet who "miss-sees" the color when looking through the screen will continue to "miss-see" the color when (still looking through the screen) the puppet is told the correct color label. Thus, they realized that a puppet who looks at an object through a red screen would continue to see the object as red even after being told the object was really yellow.

In contrast, children believed that the puppet who "miss-sees" the color because of colorblindness will "see" the color correctly when told the correct color label. Thus, they believed that a colorblind puppet who saw a red car as gray would be able to see it as red if simply told that the car was red. Even the oldest group of children proved unable to grasp that one cannot alter a colorblind puppet's subjective perception by means of verbal information. Children's failure on the Labels Question in the Colorblind condition demonstrates a failure to understand the "encapsulated" nature of color perception, a failure that persists at least through age 6.8, the oldest group tested here. This result demonstrates that children as old as 6.8 don't understand that when it comes to object appearance each person's visual perception is unique and that the information visual perception provides prevails over verbal information when forming a belief on appearance. It is possible that children, not fully understanding the colorblind puppet's visual percept equate it to a belief and hence believe that it can be altered by new verbal information. In the Screen condition, the child does not appear to have this confusion. This may be due to the fact that in the screen situation, everything is external. The child thus realizes that the visual percept yielded by looking through the colored screen is not the same as a belief, and hence that it cannot be altered by verbal information.

When we offered children the choice of labels vs. a better way to help the puppet see the color of the object correctly, most chose the better option. In the Screen condition, the better option was to take away the screen; in the Colorblind condition, it was to fix the puppet's eyes. Thus, while children of all ages tested here wrongly believed that verbal labels could alter subjective perception of color for a colorblind puppet, when given a choice of labels vs. fixing the eyes, they realized that fixing the eyes was the best way to get the puppet to see the colors correctly.

However, when children are asked whether they can align another's color perception with their own by providing them with color labels, they agree that this is indeed possible. We conclude that even children as old as 6.8 incorrectly believe that verbal information can alter visual perception. Thus, even as children enter grade school, an understanding of visual perception with its properties of subjectivity and independence from verbal information is not yet complete.

The Colorblind condition poses the greater challenge to the child's developing 
understanding of the nature of visual perception. The Screen condition may be less challenging because the child is given the experience of looking through the screen and seeing how the screen alters how colors appear. In the Colorblind condition the screen is "inside the viewer", and the child has no direct experience of this. Because the changed perception is not a change that can be ascribed to an external object (the screen), children must think of vision as a wholly subjective phenomenon, decoupled from the physical qualities of any object. Thus the subjective feature of vision is maximally expressed in the colorblind case. So it is in this condition that a full understanding of the subjective nature of color experience is best tested. It is only this condition that reveals that children do not understand that although verbal information is an originator of belief, it cannot alter perception because the mind gives primacy to visual perception over verbal information in shaping a belief about an object's appearance.

\section{REFERENCES}

Flavell, J. H., Green, F. L., \& Flavell, E. R. 1986. Development of knowledge about the Appearance-Reality Distinction. Monographs of the Society for Research in Child Development, 51 (1, Serial No. 212), $1-68$.

Gopnik, A., \& Graf, P. 1988. Knowing how you know: Young children's ability to identify and remember the sources of their beliefs. Child Development, 59, 1366-1371.

O’Neill, D. K., Astington, J., \& Flavell, J. 1992. Young children's understanding of the role that sensory experiences play in knowledge acquisition. Child Development, 63, 474-490.

Perner, J. 1992. Developing semantics for theories of mind: From propositional attitudes to mental representation. In J. W. Astington, P. L. Harris, \& D. R. Olson (Eds.), Developing theories of mind (pp. 141-172). New York: Cambridge University Press.

Pratt, C., \& Bryant, P. 1990. Young children understand that looking leads to knowing (so long as they are looking into a single barrel). Child Development, 61, 973-982.

Taylor, M., \& Flavell, J. H. 1984. Seeing and believing: Children's understanding of the distinction between appearance and reality. Child Development, 55, 1710-1720.

Wimmer, H., Hogrefe, G. J., \& Perner, J. 1988. Children's understanding of informational access as sources of knowledge. Child Development, 59, 386-396.

Wimmer, H., Hogrefe, J., \& Sodian B. 1992. A second stage in children's conception of mental life: Understanding informational accesses as origins of knowledge and belief. In J. W. Astington, P. L. Harris, \& D. R. Olson (Eds.), Developing theories of mind (pp. 173-192). New York: Cambridge University Press. 